\title{
ESTIMATIVA DA PREVALÊNCIA DE INFECCÇÕES MICOBACTE:- RIANAS EM ESCOLARES NA GUANABARA, ATRAVÉS DE TESTES INTFIADÉRMICOS COM PPD-Rt 23 E PPD-B *
}

\author{
Eliana Dessaune Madeira Christovão** e Paulo Pinto Gontijo Filho **
}

Foram testados 543 escolares do Estado da Guanabara, Brasil, na faixa etária de 12 a 14 anos, quanto d̀ hipersensibilidade tuberculínica ao PPD-Rt 23 e ao $P P D-B$.

Confirmou-se a alta prevalência de sensibilidade às tuberculoproteinas em crianças, obtendo-se $33,65 \%$ de reatores fortes ao PPD-Rt 23. Da população estudada $85,63 \%$ reagiu ao $P P D-B$, sendo que $6,26 \%$ apresentou reação maior a esta tuberculina que ao PPD-Rt 23, sugerindo uma hipersensibilização pelo bacilo Battey, ou outra microbactéria mais relacionada antigenicamente a esse microrganismo que ao Mycobacterium tuberculosis.

\section{INTRODUÇÃO}

Atualmente, o teste tuberculínico preconizado é o quantitativo, sendo as reaçôes iguais ou superiores a $10 \mathrm{~mm}$ consideradas como provocadas por infecção pelo Mycobacterium tuberculosis. Entretanto, evidências experimentais, provenientes de estudos epidemiológicos em animais de laboratório, indicam que muitas das reações fracas (5-9 mm) são conseqüência de reações cruzadas, ocasionadas por infecções subclínicas com outras micobactérias, usualmente referidas como micobactérias atípicas $(1,7,14,16,22,23,27,28)$. Sugere-se que tais reações, inespecíficas ou heterólogas, sejam mais prevalentes em regiões tropicais e subtropicais $(5,6,13,30,36)$.

No presente trabalho, foi verificada a prevalência de reatores ao PPD-B (tuberculina preparada de bacilo Battey) entre escolares do Estado da Guanabara. A escolha dessa tuberculina deveu-se ao fato das miccbactérias dos grupos II (PPD-G) e III (PPD-B) de Runyon serem os microrganismos mais freqüentemente implicados na hipersensibilização humana (8).

\section{MATERIAL E MÉTODOS}

Seiscentas e onze crianças, de 12 a 14 anos, cia rede escolar do Estado da Guanabara, foram submetidas ao teste tuberculínico diferencial, com os seguintes antígenos:

PPD-Rt 23 de $M$. tuberculosis, fornecido pelo Statens Seruminstitut de Copenhagem.

PPD-B de bacilo Battey, fornecido pela Dra. Lydia Eciwards do U.S.A. Public Health Service.

A técnica de inoculação intradérmica (Mantoux) empregada foi a preconizada pela O.M.S. e adotada pela Divisão Nacional de Tuberculose $(10,24)$.

* Trabalho realizado no Departamento de Microbiologia Médica do Instituto de Microbiologia da UFRJ, com auxilio do CEPG da UFRJ e CNPq.

* Auxiliar de Ensino do Instituto de Microbiologia da UFRJ.

** Professor Assistente do Instituto de Microbiologia da Uir.rJ.

Recebido para publicação em 10-4-1975. 
Foram excluidas as crianças com histórico de tuberculose, outras doenças crônicas, bem como as vacinadas com BCG intradérmico, o que foi verificado mediante a presença da marca característica na regiāo deltoidiana.

Foram utilizadas seringas tuberculínicas de $1 \mathrm{ml}$, marca Omega, graduadas em $0,01 \mathrm{ml}$, previamente testadas quanto ao vazamento $(9,19)$ e agulhas n. ${ }^{\circ} 26$. Todo o material foi codificado com cores diferentes e utilizado sempre para a mesma tuberculina no ciecorrer do trabalho.

Cada criança foi inoculada com PPD na face anterior de cada antebraço. Todas, portanto, receberam os dois antígenos, distribuídos ao acaso, no volume de $0,1 \mathrm{ml}$, equivalente a 2 UT do PPD-Rt 23 e 5 UT do PPD-B, respectivamente.

O diâmetro máximo transverso da enduração foi medido em milímetros, 72 horas após a inoculação. O leitor desconhecia qual das duas reaçōes estava sendo lida. Todos os testes foram procedidos e lidos pela mesma pessioa, treinada e aferida, pela Divisão Nacional de Tuberculose.

Foram considerados reatores os indivíduos que apresentaram reações iguais ou superiores a $2 \mathrm{~mm}(15,28)$.

\section{RESULTADOS}

Das 611 crianças testadas apresentaram-se para leitura $543(88,87 \%)$. Todas eram resicientes permanentes da Guanabara, sendo a maioria natural do Grande Rio, nunca tendo residido em outra área geográfica.

As características da população testada encontram-se na Tabela I.

\section{TABELA. I}

Caracteristica cả população escolar testada.

\begin{tabular}{l|lc}
\hline Sexo & & $\begin{array}{c}\text { Porcenta- } \\
\text { gem \% }\end{array}$ \\
\hline & Masculino & 50,09 \\
& Feminino & 49,91 \\
\hline Cor & Branca & 66,91 \\
& Parda & $2,1,19$ \\
& Preta & 11,90 \\
\hline
\end{tabular}

$\mathrm{Na}$ distribuição das reações aos dois antígenos, se observaram diferenças significativas com relação ao sexo (Tabela II). Pôde-se notar uma marcante predominância de reações maiores ao PPD-Rt 23 que ao PPD-B, bem como de reações idênticas aos do1s antígenos. Houve $33,65 \%$ de reatores fortes ( $>10 \mathrm{~mm}$ ) ao PPD-Rt 23 .

Considerancio-se como desprezíveis as cifferenças de até $2 \mathrm{~mm}$ entre as reações observadas aos dois PPDs, em um mesmo indivíduo (31), temos $40,07 \%$ de reaçōes idênticas entre os meninos e $43,18 \%$ entre as meninas, $43,75 \%$ do sexo masculino e $39,48 \%$ do feminino apresentaram reações maiores ao PPD-Rt 23 enquanto $6,25 \%$ e $6,27 \%$ entre meninos e meninas, respectivamente, mostraram reações maiores ao PPD-B (Tabela II) .

A proporção de meninas que não apresentam reação ao PPD-Rt 23 foi ligeiramente maior que a dos meninos $(14,76 \%$ e $13,60 \%$ respectivamente), o mesmo ocorrencio com relação ao $\mathrm{PPD}-\mathrm{B}$, onde $18,08 \%$ e $10,66 \%$ das meninas e meninos não reagiram.

A reação média ao $P P D-B \quad(4,32 \mathrm{~mm})$ foi inferior àquela apresentada para o Rt $23(6,16 \mathrm{~mm})$. As reaçōes a este último PPD, na amostragem testada, forneceram uma curva bimodal, ao contrário do que se cbserva com a curva do PPD-B. (Fig. I)

\section{DISCUSSÃO}

Inúmeras evidências fornecidas por in. quéritos epiciemiológicos utilizando-se tuberculinas (PPLS) preparadas de diferentes amostras de micobactérias e de estudos em cobaics, indicam que infecçōes causadas por outras micobactérias (micobactérias atípicas) que não o bacilo da tuberculose são comuns e acarretam reações cruzadas no teste tuberculínico (15).

Pcr cutro lado, o emprego da vacina BCG na imunização contra a tuberculose, tem fornecido resultados contrastantes em diferentes áreas geográficas. Uma das explicações para o fato, é que as diferenças sejam devidas a infecções subclínicas com micobactérias atípicas. Infecções com esses microrganismes constituiriam, portanto, um tipo de vacinação natural que se assemelha à vacinação BCG (5). O conheci. mento destas infecçōes é ainda limitado, embora estudos epiciemiológicos em animais de laboratório demonstrem que infec- 
Tabela II

Relaçăo entre o tamanho das reações ao PPD-Rt 23 e ao PPD-B em escolares

\begin{tabular}{|c|c|c|c|c|c|c|}
\hline GRUPO & \multicolumn{2}{|c|}{$\begin{array}{l}\text { Masculino } \\
\mathrm{N}^{\mathrm{o}} \%\end{array}$} & \multicolumn{2}{|c|}{$\begin{array}{c}\text { Feminino } \\
\text { No } \%\end{array}$} & \multicolumn{2}{|c|}{$\begin{array}{l}\text { Total } \\
\text { No } \%\end{array}$} \\
\hline $\begin{array}{l}\text { 1) Reações maiores ao PPD-RT } \\
23 \text { que ao PPD-B com diferen- } \\
\text { ça }>6 \mathrm{~mm} \ldots \ldots \ldots \ldots \ldots \ldots\end{array}$ & 55 & 20,22 & 61 & 22,51 & 116 & 21,37 \\
\hline $\begin{array}{l}\text { 2) Reaçōes maiores ao PDD-Rt } 23 \\
\text { que ao PPD-B com diferença } \\
\text { de } 3-5 \mathrm{~mm} \ldots \ldots \ldots \ldots \ldots \ldots\end{array}$ & 64 & 23,53 & 46 & 16,98 & 110 & 20,25 \\
\hline TOTAL GRUFOS 1 e $2 \ldots \ldots \ldots$ & 119 & 43,75 & 107 & 39,49 & 2.26 & 11,62 \\
\hline 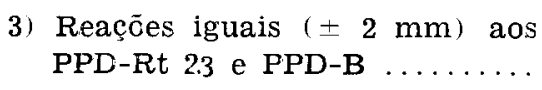 & 109 & 40,07 & 117 & 43,17 & 226 & 11,62 \\
\hline $\begin{array}{l}\text { 4) Reações maiores ao PPD-B que } \\
\text { ao PPD-Rt } 23 \text { com diferen- } \\
\text { ça } \$ 6 \mathrm{~mm} \ldots \ldots \ldots \ldots \ldots \ldots\end{array}$ & 3 & 1,10 & 2 & 0.74 & 5 & 0.92 \\
\hline $\begin{array}{l}\text { 5) Reaçōes maicres ao } \mathrm{PPD}-\mathrm{B} \text { que } \\
\text { ao } \mathrm{PPD}-\mathrm{Rt} 23 \text { com diferença de } \\
3-6 \mathrm{~mm} \ldots \ldots \ldots \ldots \ldots \ldots \ldots\end{array}$ & 14 & 5,15 & 15 & 5,53 & $2 \subseteq$ & 5,34 \\
\hline TOTAL GRUPOS 4 e $5 \ldots \ldots \ldots$ & 17 & 8,25 & 17 & 6,27 & 34 & 6,26 \\
\hline Ausência de reações aos 2 PPDs .. & 27 & 9.93 & 30 & 11,07 & 5.7 & 10,50 \\
\hline TCTAL & 272 & 100 & 271 & 100 & 543 & 100 \\
\hline
\end{tabular}

ções com esses microorganismos induzem, com freqüência e intensidade diferentes, reações tuberculínicas inespecíficas $(1,7$, $14,16,22,23,27,28$ ). Adicionalmente, outros estudos laboratoriais e inquéritos epidemiológicos sugerem que infecções com micobactérias atipicas criam alguma capaciade, extremamente variável entre as diferentes espécies, de modificar o curso da tuberculose doença $(12,20,21,26,29,32,33$, 34, 35).
As micobactérias dos Grupos II (escotocromogênicas de crescimento lento) e III (não cromogênicas de crescimento lento) de Runyon, são as mais freqüentemente implicadas na hipersensibilidade humana $(8,15)$. Até o momento, a maioria dos testes $_{S}$ realizados no homem tem sido com PPD-B, preparado a partir de uma amostra do grupo III de Runyon (Bacilo Battey), isolada de um paciente com pneumopatia. Essa tuberculina é portanto reco- 


\section{DISTRIBUICĀO DE FREQUÉNCIA DAS REÁAC̄EES}

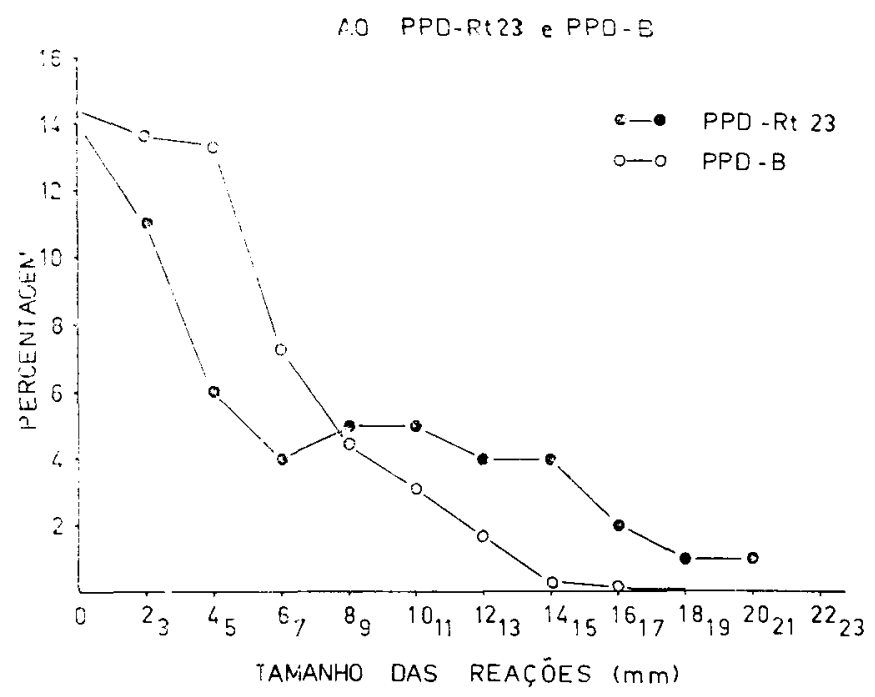

Fig. I - Distribuição de Freqütencia das Reações ao PPD-Rt 23 e PPD-B em Escolares da Guanabara, Brasil.

mendada atualmente para uso no teste cutâneo comparativo (4).

Comstock et al (11) compararam as tuberculinas PPD-Rt 23 (do Statens Seruminstitut, distribuda pela OMS) e PPD-S (preparada de $M$. tuberculosis e padrão nos EEUU), em indivíduos com hipersensilidade tuberculínica específica, ou seja, infectados com $M$. tuberculosis, e em indivíduos cuja hipersensibilidade parece ter resultado de infecções subclínicas com $M$. intracellulare (Bacilo Battey). No primeiro grupo, 2.5 UT de PPD-Rt 23 foram praticamente equivalentes a 5 UT de PPD-S, enquanto no segundo provocaram consideravelmente mais reaçōes que as 5 UT de PPD-S. Portanto, onde a hipersensibilidade inespecifica é mais freqüente que a específica, há tenđência do PPD-Rt 23 de provocar mais reaçōes, o que pode tornar inconveniente seu emprego, isto possivelmente devido ao PPD-Rt 23 ser um produto menos purificado que o PPD-S.

Vários trabalhos evidenciam que reaçōes cruzadas são mais freqüentes em regiōes tropicais e subtropicais $(5,6,13,30$, 36). No Brasil, evidências nesse sentido foram observadas por Almeida (3), que verificou uma maior porcentagem de reatores fracos ao PPD-Rt 2.3 no Norte $(8,7 \%$ em Manaus) do que no Sul $(0,8 \%$ em Curiti- ba). No Nordeste, Estado do Ceará Façanha \& Codes (17) e Façanha et al (18) utilizando PPD-G (de $M$. scrofulaceum, amostra Gause) demonstraram uma maior porcentagem de reatores entre recrutas $e$ escolares, a esta tuberlina que ao PPD-Rt 23.

No presente inquérito a maioria das crianças reagiu aos dois antigenos testados. Considerando-se as reações superiores a $2 \mathrm{~mm}$ como positivas, $85,63 \%$ das crianças testadas reagiram ao $\mathrm{PPD}-\mathrm{B}$, confirmando as observações já citadas realizadas em outras áreas tropicais, enquanto $85,82 \%$ reagiram ao PPD-Rt 23.

O problema da interpretação dos resultados obtidos com duas tuberculinas é o mesmo encontrado quando da utilização da tuberculina padrão, qual seja a distinção entre reações especificas e cruzadas.

Sabe-se de estudos experimentais em cobaias (14), que ocorre grande hipersensibilização cruzada entre as várias infecções micobacterianas. Conseqüentemente, não se pode concluir que a hipersensibilidade a esses antígenos (PPD-Rt 23 e PPD-B) indique infecção, passada ou presente, com o microrganismo a partir do qual foi preparado o PPD.

A hipersensibilidade cruzada à tuberculina padrão, induzida por infecção com 
micobactéria atípica é caracteristicamente mais fraca que a hipersensibilidade induzida pela infecção tuberculosa (2). Assim, a distinção entre a maioria das reações cruzadas e a maioria das reações específicas pode ser feita pelo uso do teste tuberculínico quantitativo diferencial, medindo-se o grau de hipersensibilidade, em vez de um teste qualitativo para determinar simplesmente a presença ou ausência de reação. Levando-se em conta este critério, 6,26\% da população estudada apresentou reaçóes maiores ao PPD-B, sugerindo uma hipersensibilização pelo Bacilo Battey ou por outra micobactéria mais relacionada antigênicamente a este microrganismo que as Mycobacterium tuberculosis.

Edwards (15), encontrou um tamanho médio para as reações cutâneas ao PPD-S e PPD-B de $10,3 \mathrm{~mm}$ e $7,7 \mathrm{~mm}$, respectivamente, quando de testes em mais de ...... 200.000 recrutas nos EEUU. No presente trabalho o tamanho médio das reações ao PPD-Rt 23 foi de $6,16 \mathrm{~mm}$ e ao PPD-B de $4,32 \mathrm{~mm}$.
A curva apresentada pelas reações ao PPD-Rt 23 não é muito diferente daquela apresentada por paciente tuberculosos (25), exceto quanto à predominância de reações fracas (Fig. 1).

O PPD-B apresentou uma curva diferente, onde se evidencia a maior freqüência de reatores fracos a essa tuberculina que ao PPD-Rt 23. A maioria das reações induzidas pelo PPD-B foram fracas (2-6 $\mathrm{mm}$ ), enquanto a maior parte das reações maiores ou iguais a $10 \mathrm{~mm}$ deveram-se ao PPD-Rt 23 (Fig. 1).

\section{AGRADECIMENTOS}

Os autores agradecem a Dra. Lydia Ed'wards, do U.S.A. Public Health Service o fornecimento do PPD-B. Agradecem à Divisão Nacional de Tuberculose o material e treinamento para a aplicação dos testes, bem como à Coordenação Geral de Saúcie Pública do Estado da Guanabara pela autorização para utilizar escolares da rede oficial de ensino.

\section{SUMMARY}

The tubberculin hypersensitivity to PPD-Rt 23 and PPD-B was tested on 543 children, 12 to 14 years old in "Estado da Guanabara", Braiil.

The sensitivity to tuberculin proteins was found high. About $33,65 \%$ of the children showed strong reactions to PPD-Rt 23 in about $6,26 \%$ of the children population, what suggests a hypersensitivity due to the Battey bacillus or another mycobacteria more antigenically related with this microorganism than the M. tuberculosis.

\section{REFERÊNCIAS BIBLIOGRÁFICAS}

1. ABRAHAMS, E. W. \& SILVERSTONE, H. - Epidemiological evidence of the presence of non-tuberculous sensitivity to tuberculin in Queensland. $T u$ bercle 42: 487-499, 1961 .

2. ABRAHAMS, E. W. - Original Mycobacterial Sin Tubercle 51: 316-321, 1970.

3. ALMEIDA, A. P. de - Prevalência da Infecção tuberculosa em escolares das capitais brasileiras, Rev. Nac. Tuberc. 17: 155-174, 1973.

4. AMERICAN THORACIC SOCIETY The tuberculin skin test - A Statement by the Comitee on Diagnostic.
Amer. Rev. Resp. Dis. 104: 769-775, 1971.

5. BATES, L E.; BUSK, T. \& PALMER C. E. - Research contributions of BCG Vaccination Programs: II. Tuberculin Sensitivity at different altitudes of residence. Publ. Hilth. Rep. 66: 1427-1442, 1951.

6. BEUNDERS, B. J. W.; BLEIKER, M. A. \& GRIEF, W. A. - Cutaneous reactions to human and avian PPD, after BCG vaccination - Selected Papers, The Royal Netherlands Tuberculosis Association. The Hague 3.60 , quoted by British Tuberculosis Association, 1963. 
7. BJERKDAL, T. - Naturally acquired sensitivity in Norway. Acta Tuberc. Pneumol. Scand. 43: 275-298, 1963.

8. CHIN, J. - Comparative tuberculin testing in Malaya. Tubercle (London) 45: 114-124, 1964.

9. CHRISTOVÃO, E. D. M.; GONTIJO FILHO, P. P. \& CAMPOS NETO, A. Medida do vazamento de seringas tuberculínicas. Rev. Div. Nac. Tuberc. 18: $172-179,1974$.

10. COMISSĀO TÉCNICA DA C.N.C.T. Prova Tuberculínica em Saúde Pública (2a reccmendação). Rev. Div. Nac. Tuberc. 12: 2,19-230, 1968.

11. COMSTOCK, G. M.; EDWARDS, L. B.; PHILIP, R. N. \& WINN, W. A - A comparasion in U.S.A. of two tuberculins, PPD-S and PPD-Rt 23. Bull. Wld. Mlth. Org. 31: 161-170, 1964.

12. COMSTOCK, G. M. \& PALMER, C. E. - Longterm results of BCG vaccination in the Southern United States. Amer. Rev. Resp. Dis. 93: 171-183, 1966.

13. DAVIS, G. H. G. \& OGUMBI, O. Sensitivity to six mycobacterial antigens in young Nigerian adults. Bull. Wld. Hlth. Org. 36: 791-799, 1967.

14. EDWARDS, L. B.; PALMER, C. E.; AFFRONTI, L. F.; HOPWOOD, L. \& EDWARDS, P. $Q$. - Epidemiologic response to experimental infection studies of tuberculin sensitivity - II. with mycobacteria isolated from human sources. Amer. J. Hyg. 71: 218$-241,1960$.

15. EDWARDS, L. B. - Current status of the tuberculin test. Ann. New York Acad. Sci. 106: 32-42, 1963.

16. EDWARDS, L. B. \& SMITH, D. T. Community-wide tuberculin testing study in Pamlico County, North Caroline. Amer. Rev. Resp. Dis. 92: 43-54, 1965.

17. FAÇANHA, M G. F. \& CODES, F. J. M. - Sensibilidacie ao PPD-G. Arq. Bras. Tuberc. Doenç. Tórax. 27: 87-97, 1968.

18. FAÇANHA, M. G. F.; MENDES, J. A. \& CODES, F. J. M. - Infecção tuberculose em Maranguape (Ce). Arq. Bras. Tuberc. e Doenç. Tórax, 28: 41-471969 .

19. GULD, J. \& RUD,, C. - Measurement of leakage of tuberculin syringes. Brit. Med. J. 1: 368-370, 1963.

20. KLUGH, G. A. \& PRAT'T, P. C. Experimental immunivation of guinea pigs with photo-chromogenic acid-fast bacilli. Amer. Rev. Resp. Dis. 85: 78-83, 1962

21. LARSON, C. L. \& WICHT, W. C. Resistance to infection with virulent tubercle bacilli in mice immunized with viable Mycobaterium balnei and unclassified mycobacteria administered aerogenically. Amer, Rev. Resp. Dis. 88: 456-461, 1963.

22. MAGNUSSON, M.; BLEIKER, M. A. \& GRIEP, W. A. - Comparative intradermal reactions to young Dutch males to human purified tuberculin (PPD) and other sensitins. Acta Tuberc. Pneumol. Scand. 42: 53-72, 1962.

23. NISSEN-MEYER, S. - Epidemiologic of tuberculin sensitivity: III - Estimation of prevalences of mycobacterial infection from results of skin tests with mycobaterial antigens. Amer. J. Hyg. 72: 169-194, 1960.

24. OPS/OMS/CD/TB/8/ 1969.

25. PALMER, G. E. \& BATES, L. E. - Tuberculin sensitivity of tuberculous patients. Bull. Wld. Hlth. Org. 7: 171$-188,1952$

26. PALMER, C E.; SHAW, L. W. \& COMSTOCK, G. W. - Community trials of $\mathrm{BCG}$ vaccination. Amer. $\mathrm{T} u$ berc. 77: 877-907, 1958.

27. PALMER, C. E.; EDWARDS, L. B.; HOPWOOD, L. \& EDWARDS, P. Q. Experimental and epidemiologic basis for the interpretation of tuberculin skin sensitivity. J. Pediatrics 55: 413$-429,1959$

28. PALMER, C. E. \& EDWARDS, L. B. Geographic variations in the prevalence of sensitivity to tuberculin (PPD-S) and to the Battey antigen (PPD-B) throughout the United States. Bull Intern. Un. Tuberc. 32: 373$383,1962$.

29. PALMER, C E. \& HOPWOOD, L. Effect of previous infection with unclassified mycobacteria on survival of guinea pigs challenged with virulent tubercle bacilli. Bull. Intern. Un. Tuberc. 32: 398-402, 1962.

30. PINTO, M. R. M.; ARSECULERATNE, S. N.; URAGODA, C. G. \& HEMAWARDENE, D. M. - Differential tuberculin testing in rural populations in Ceylon. Tubercle 53: 182-197, 1972.

31. PINTO, M. R. M.; ARSECULERATNE, S. N.; URAGODA, C. G. \& HEMAWARDENE, D. M - The differential tuberculin test in tuberculosis patients Tubercle 54: 46-56, 1973. 